\title{
Energy Efficient Battery Discharge Rate Based Asynchronous Duty Cycling Protocol for WSN to improve Network Lifetime
}

\author{
Amandeep Kaur ${ }^{1}$, Dr. Rajneesh Gujral ${ }^{2}$ \\ ${ }^{1,2}$ (Department of Computer Science and Engineering, Maharishi Markandeswar University,Mullana, India)
}

\begin{abstract}
In Energy Constrained Wireless Sensor Network, maximizing network lifetime is an important issue. The challenge is to find out the optimal schedule of duty cycle for nodes while maintaining the sufficient number of active nodes for network coverage. In this paper we propose the Energy Efficient Battery Discharge Rate Based (E $\left.E^{2} B D R D C\right)$ Asynchronous Duty Cycling Protocol for WSN which consider the battery discharge rate of active node to find the duty cycle of inactive nodes. Further the $E^{2} B D R D C$ protocol improves the energy consumption and network lifetime $30 \%$ and $20 \%$ as compared to DCBSP (Discharge Curve Back off Sleep Protocol).
\end{abstract}

Keywords - Duty cycle, Network Lifetime, SNs, WSN.

\section{INTRODUCTION}

As Computing become exponentially smaller and cheaper with each passing year. Semiconductor technology can be used to build mechanical structure called Sensor Node (SN) that can sense the surrounding phenomenon including temperature, vibrations and pressure. A Wireless Sensor Network (WSN) [1,2] consists of the small SNs having sensing, computing and communication capability. One of the features of WSN is that SNs are resource constrained having less battery power, limited storage capacity. SNs are normally deployed in unattended environment make it impossible to recharge or replace the battery. So the efforts must be employed to reduce the energy consumption of node thereby improving network lifetime.

However in WSN large number of SNs is deployed in sensing region so there is the probability of redundancy which would cause energy wastage. To maximize the network lifetime it is necessary to minimize the redundancy and number of active nodes while maintaining the optimal coverage. To eliminate the redundancy in WSN adaptively selects only minimum number of nodes to remain active for maintaining optimal coverage. For this purpose many energy efficient duty cycle protocol has been proposed. Duty cycling can be divided into different ways in WSN: Synchronous, Asynchronous and On-demand protocols [3]. Our major contribution is the design of energy efficient protocol for duty cycling based on battery discharge rate to improve network lifetime. In this paper active/sleep state of node is decided based on the remaining battery level.

As in DCBSP (Discharge Curve Back off Sleep Protocol) [4] [13] [14] protocol is also asynchronous MAC protocol uses the battery discharge rate of active node to decide the duty cycle of inactive nodes. In DCBSP inactive nodes wake up only when the current active node is close to the dead state. If more event occurs at the time of active node duration [15] [16] node may dead before its predefined time causes less reliable network and reduction in network lifetime if less event occurs. So $E^{2} B D R D C$ protocol improves the network reliability and network lifetime by considering these [17] [18] factors.

The Rest of the paper is organized as follows: Related Work is discussed in section II. Section III elaborates the $E^{2} B D R D C$ protocol and Simulation results are discussed in Section IV. Conclusion and future work is presented in Section V.

\section{RELATED WORK}

We discuss the some existing energy efficient protocol for duty cycle in this section. Many Sleep-wakes scheduling protocol is proposed in literature. Synchronized protocols, such as S-MAC [5] and T-MAC [6], decide a schedule that specifies when nodes are wake and sleep within a slot. Specifying the slot when nodes must be wake in order to communicate reduces the time and energy wasted in idle listening. In synchronous scheduling protocol [7-9] synchronization information is exchange periodically among neighbours causes message overhead. Asynchronous protocols such as B-MAC [10], and WiseMAC [11], X-MAC [12] rely on low power listening (LPL), also called preamble sampling. Idle listening is reduced in asynchronous protocols by shifting the burden of synchronization to the sender. When a sender has data, the sender transmits a preamble 
that is at least as long as the sleep period of the receiver. The receiver wake up, detect the preamble, and stay wake to receive the data. In asynchronous scheduling protocols node wakes up independently of its neighbours. In Random Back off Sleep Protocol (RBSP) [13], neighbouring node wakes up very frequently when the remaining energy of active node very less. DCBSP (Discharge Curve Back off Sleep Protocol) protocol uses the battery discharge rate of active node to decide the duty cycle of inactive nodes. In DCBSP inactive nodes wake up only when the current active node is close to the dead state which leads to the reduction in network lifetime. In $E^{2} B D R D C$ asynchronous duty cycling protocol one of the neighbour node goes to active state when the battery discharge rate of current active node remain $50 \%$ to improve network lifetime.

\section{PROPOSED PROTOCOL}

Before describing $E^{2} B D R D C$ asynchronous duty cycling protocol following assumptions are considered:

1. SNs are randomly distributed within a circular field.

2. BS is located at centre of sensing area.

3. SNs and the BS are stationary after deployment.

4. SNs are location-aware. The location of the BS is known by each node.

5. All SNs are homogeneous, i.e., they have the same energy.

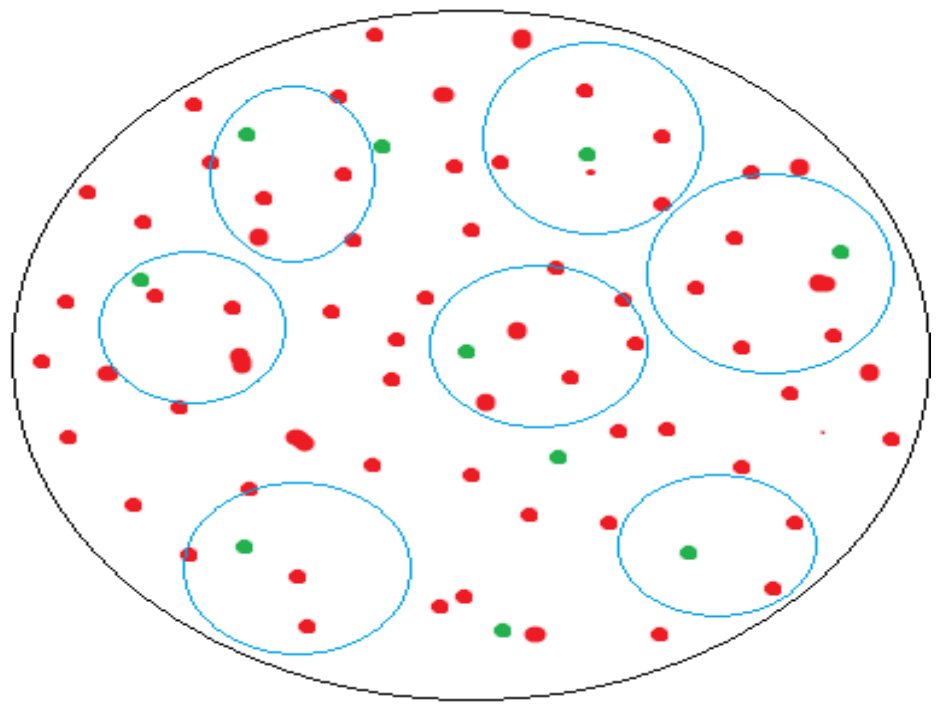

Fig. 1: WSN Topology: Active/Sleep Nodes

During set up phase some SNs are uniformly selected as active node (green colour) in the network and rest in sleep state (red colour). [19-22] Fixed duration $\mathrm{T}$ is associated with each active node during first round. When the duration of node expires it broadcast the packet having its remaining battery level B to its neighbours. All neighbour nodes of active node goes to sleep state till next duration and any one of the neighbour wake up only if the battery percentage of current active node is less than 50\%.If more event occurs during active duration then active node can go to dead state before $\mathrm{T}$ sec. When the battery level of active node remains less than $50 \%$ before $\mathrm{T}$ sec, it broadcast the packet to its neighbour to select next active node for remaining duration. For example node 2 in Fig. 2 is in active state and its fixed duration is $\mathrm{T}=5 \mathrm{sec}$. When the duration of node expires it broadcast a packet having its current battery level i.e. $4 \%$ value to its neighbours. Node 4 will be active and node 1,2and 3 goes to sleep state for next $5 \mathrm{sec}$ to uniformly distributed the active/sleep pattern among all neighbours as in Fig. 3. 


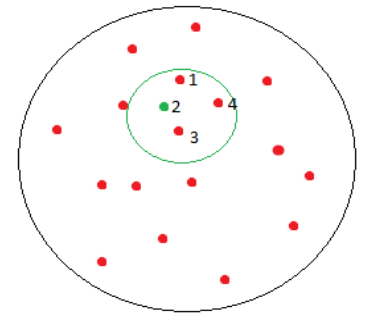

Fig. 2: Sleep/Active State during T sec

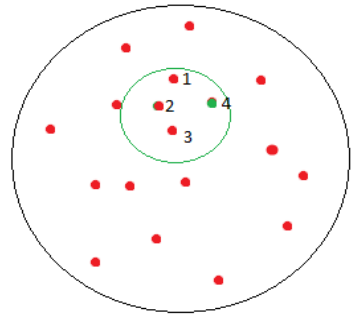

Fig. 3: Sleep/Active State after T sec

Scheduling mechanism of $E^{2} B D R D C$ and DCBSP protocol is shown in Fig. 4 and Fig. 5. Time is divided into slots of $\mathrm{T}$ sec. Node A wakes up for its duration and send beacon packet to node B containing its battery status. Node B wake up only if the battery level is less than 50\%. [23-25] If the battery level of node A goes less than $10 \%$ before $\mathrm{T}$ sec it send packet to Node B to get active for next duration increasing availability of network as in DCBSP protocol node A send packet to node B after T sec only causing network in-availability because of both nodes goes to sleep state simultaneously as in Fig. 5.

A
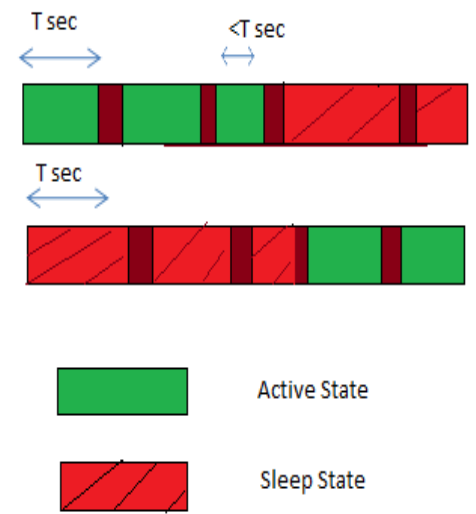

Beacon Packet

Timing Diagram:Proposed Protocol

Fig. 4: Scheduling Mechanism: $E^{2} B D R D C$ Protocol
A
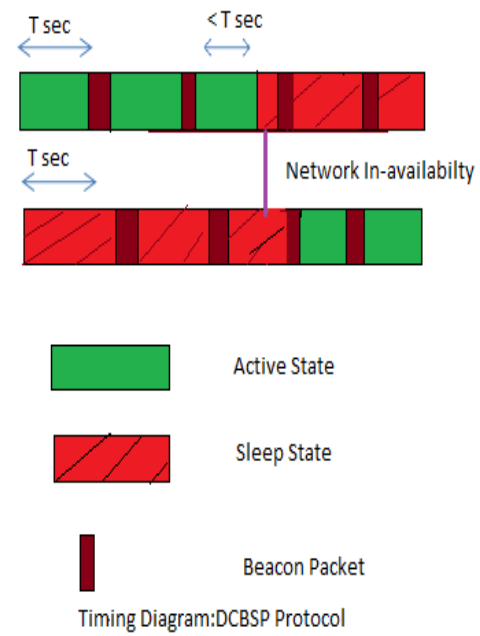

Fig. 5: Scheduling Mechanism: DCBSP Protocol

\subsection{Energy Model}

$E^{2} B D R D C$ asynchronous duty cycling protocol uses energy model discussed in [15].To transmit an L-bit data to a distance $d$, the radio expands energy.

$$
\begin{aligned}
& E_{T X}(L, d)=L * E_{\text {elec }}+L * \in_{f s} * d^{4} \text { if } d \geq d_{0} \\
& L * E_{\text {elec }}+L^{*} \in_{f s} * d^{2} \text { if } d \prec d_{0}
\end{aligned}
$$


where $E_{T X}(L, d)$ is energy consumed by a node to transmit $\mathrm{L}$ bit packet at distance $\mathrm{D}, E_{\text {elec }}$ is the electronic energy that depends on factors such as the digital coding, coding, modulation or, is the amplifier energy that depends on the transmission distance and the acceptable bit-error rate.

While receiving, the radio expands energy:

$$
E_{R X}=L * E_{\text {elec }}
$$

$E_{R X}(L)$ is energy dissipated by a node to received $\mathrm{L}$ bit packet .

\section{Simulation AND RESUlTS}

In this section we evaluate the performance of $E^{2} B D R D C$ asynchronous duty cycling protocol. Simulation model is designed using MALTAB. We assume network of $200 * 200$ meters $^{2}$ and number of nodes varies from 100-500.Intial energy of node is 0.5 Joules. Performance metrics considered are network lifetime, average number of active nodes and average energy consumption $E^{2} B D R D C$ protocol is compared with DCBSP protocol and simulation results shows that proposed protocol improves the network lifetime and overall energy consumption of network 10-20\% as compared to DCBSP protocol. Network lifetime of $E^{2} B D R D C$ and DCBSP protocol is shown in Fig. 6.We Compare the network lifetime in terms of number of dead nodes. As shown in Fig. $8 E^{2} B D R D C$ asynchronous duty cycling protocol extends the network lifetime by decreasing the number of dead nodes as compared to DCBSP protocol. Fig. 6 shows the average energy comparison of the approaches. Average energy consumption per simulation is less in $E^{2} B D R D C$ protocol due to availability of active node all times in $E^{2} B D R D C$ protocol. Total number of active nodes in each simulation for both approaches is shown in Fig. 7.

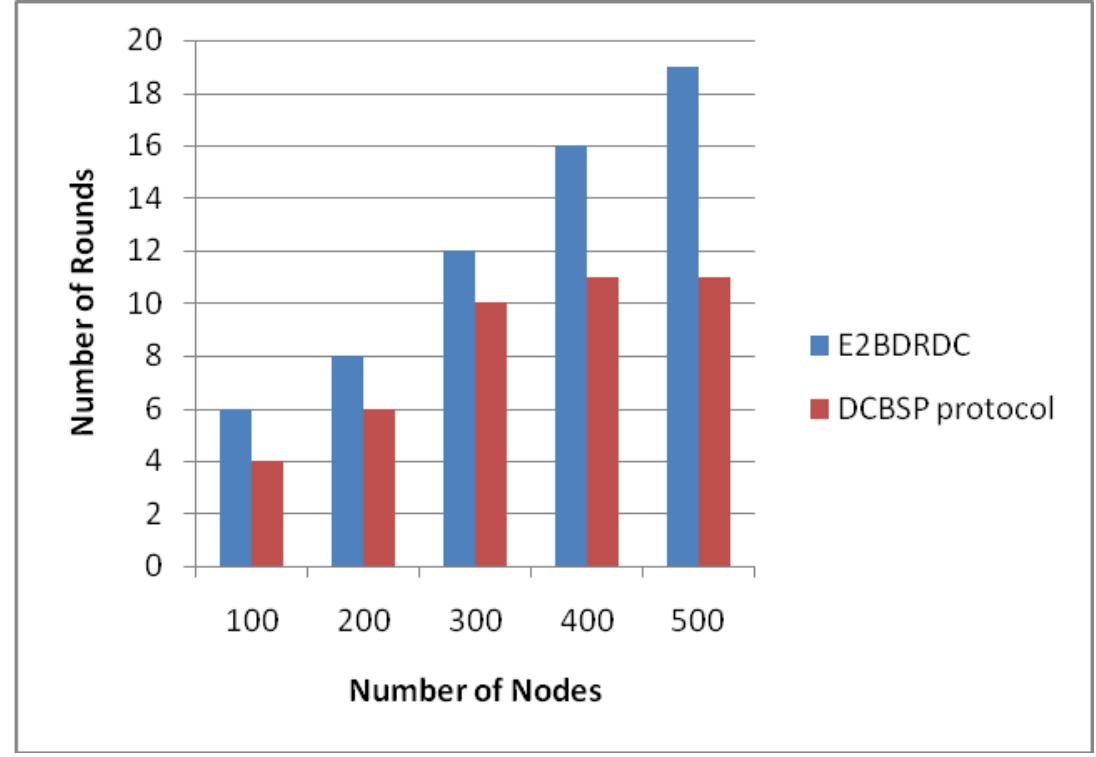

Fig. 6: Network Lifetime 


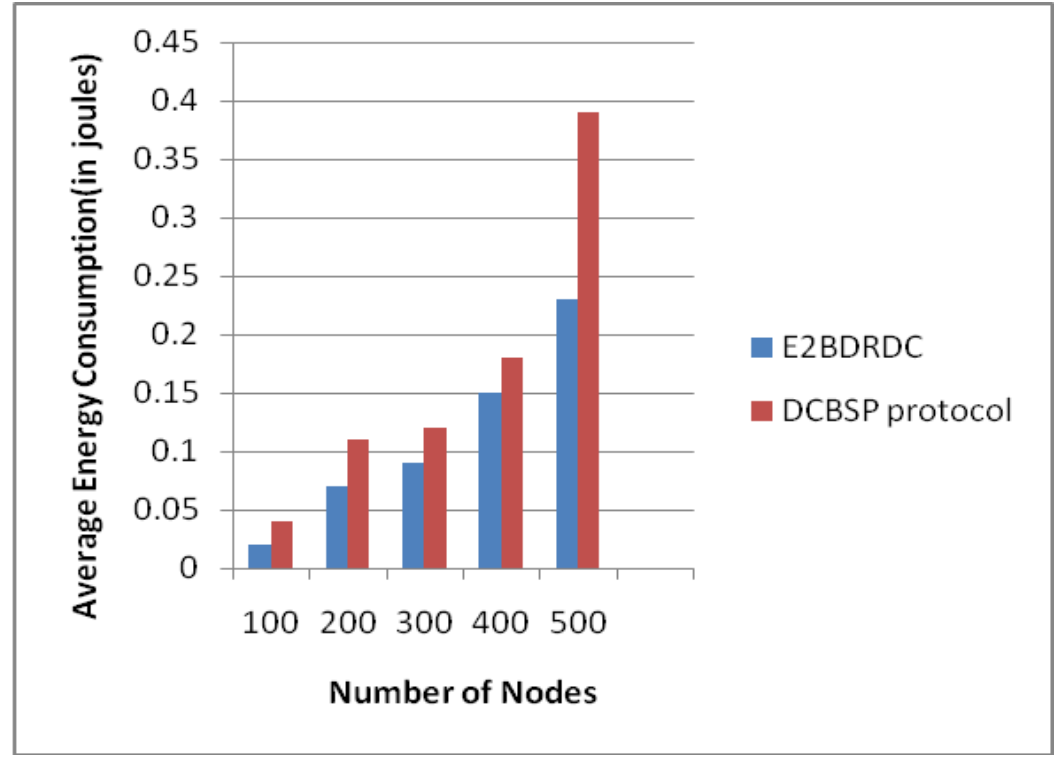

Fig. 7: Average Energy Consumption

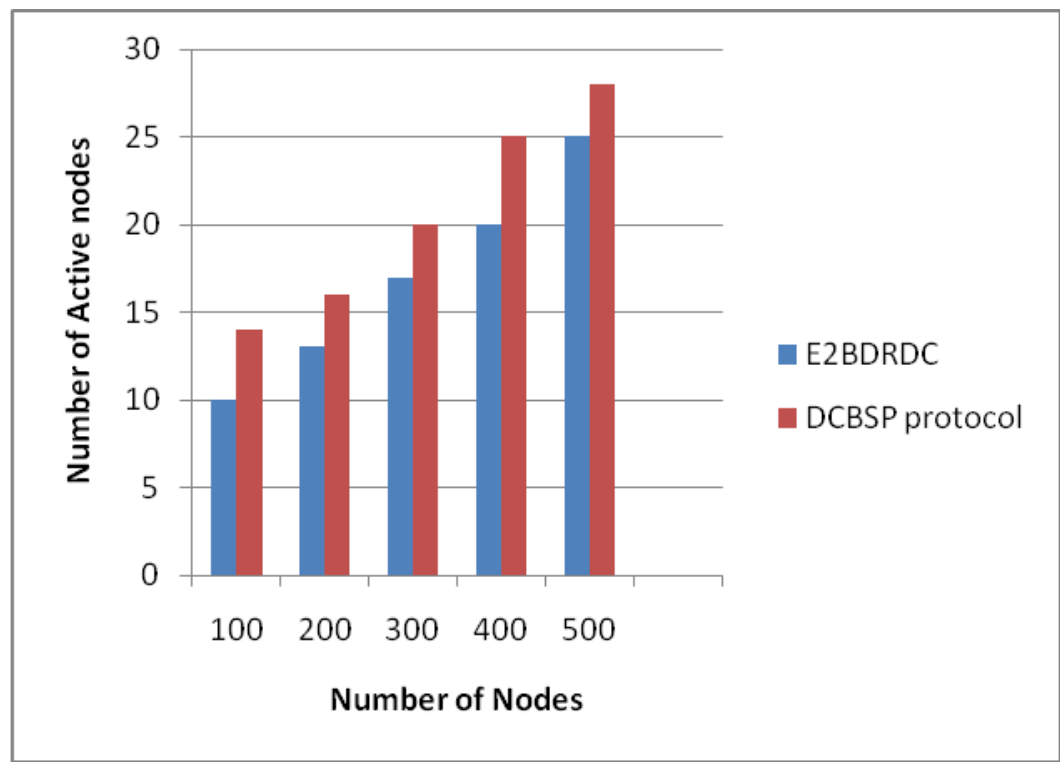

Fig. 8: Number of Active Nodes

\section{CONCLUSION AND FUTURE WORK}

We have proposed the Energy Efficient Battery Discharge Rate Based Asynchronous Duty Cycling Protocol $\left(E^{2} B D R D C\right)$ in which sleep nodes goes to active state after predefine time slot to improve Network Lifetime. Proposed protocol also avoid unnecessary frequent wake up of sleeping nodes as in existing protocols. In proposed protocol sleep node goes to active state immediately if any active node dead before its active duration to improve the network reliability. Further we extend our proposed protocol to increase the network connectivity.

\section{REFERENCES}

[1] Akyildiz, W. Su, Y. Sankarasubramaniam, E. Cayirici, Wireless sensor networks: A survey, Elsevier, Computer Networks, vol. 38 (4,) 2002, 393-422. 
[2] Ghosh, S. Das, Coverage and connectivity issues in wireless sensor networks: A survey, Science Direct, Pervasive and Mobile Computing 4 (2008), 303-334.

[3] G. Anastasi, M. Conti, M. Di Francesco, A. Passarella, Energy Conservation in Wireless Sensor Networks: a Survey, Ad Hoc Networks, vol. 7(3), May 2009.

[4] Avinash More,, Vijay Raisinghani, Discharge Curve Backoff Sleep Protocol for Energy Efficient Coverage in Wireless Sensor Networks, in Proceedings of 3rd International Conference on Recent Trends in Computing (ICRTC), 2015, pp.1131-1139.

[5] W. Ye, J. Heidemann, and D. Estrin, An energy-efficient mac protocol for wireless sensor networks, in Proceedings of the 21st International Annual Joint Conference of the IEEE Computer and Communications Societies (INFOCOM 2002), Newyork-USA.

[6] T. van Dam and K. Langendoen, An Adaptive Energy-efficient MAC Protocol for Wireless Sensor Networks, in ACM SenSys, 2003, pp. 171-180.

[7] W. Ye, J. Heidemann, and D. Estrin, Medium Access Control with Coordinated Adaptive Sleeping for Wireless Sensor Networks, IEEE/ACM Transactions on Networking, vol. 12(3), pp. 493 - 506, June 2004.

[8] G. Lu, B. Krishnamachari, and C. Raghavendra, An adaptive energy-efficient and low-latency mac for data gathering in wireless sensor networks, in International Parallel and Distributed Processing Symposium , 2004.

[9] R. Jurdak, P. Baldi and C. V. Lopes, Adaptive Low Power Listening for Wireless Sensor Networks, Transactions on Mobile Computing Vol. 6 (8), pp. 988-1004, Aug. 2007.

[10] Jitender Grover, Shikha Sharma and Mohit Sharma, "Reliable SPIN in Wireless Sensor Network: A Review", IOSR Journal of Computer Engineering (IOSR-JCE), ISSN: 2278-0661, Vol. 16, Issue 6(III), DOI: 10.9790/0661-16637983, pp. 79-83, Nov.-Dec. 2014.

[11] Jitender Grover, Shikha Sharma and Mohit Sharma, "A Study of Geographic Adaptive Fidelity Routing Protocol in Wireless Sensor Network", IOSR Journal of Computer Engineering (IOSR-JCE), ISSN: 2278-0661, Volume 16, Issue 5, Ver. IV, DOI: 10.9790/0661-16548896, pp. 88-96, Sep-Oct. 2014. (I.F. 1.686)

[12] Sandeep Singh, Jitender Grover, Randeep Singh Chib, "Review Study of Wireless Sensor Networks, Architecture and Design Issues Related to WSN", Journal of Engineering Research and Studies, Vol. IV, Issue I, Jan.-March, 2014.

[13] Jitender Grover and Shivangi Katiyar, "Agent Based Dynamic Load Balancing in Cloud Computing", IEEE International Conference on Human Computer Interactions (ICHCI'13), Saveetha University, Chennai, DOI: 10.1109/ICHCIIEEE.2013.6887799, pp. 1-6, 23-24, August 2013.

[14] J. Polastre, J. Hill, and D. Culler, Versatile low power media access for wireless sensor networks, in The Second ACM Conference on Embedded Networked Sensor Systems (SenSys), pp. 95-107, November 2004.

[15] Jitender Grover \& Reena Rani, "Probabilistic Density Based Adaptive Clustering Scheme to Improve Network Survivability in WSN", IEEE Fifth International Conference on Computing, Communications and Networking Technologies (ICCCNT 2014), Hefei, Anhui, China, DOI: 10.1109/ICCCNT.2014.6963132, pp. 1-7, July 11-13, 2014.

[16] Jitender Grover, Shikha and Mohit Sharma, "Cloud Computing and Its Security Issues - A Review", IEEE Fifth International Conference on Computing, Communications and Networking Technologies (ICCCNT 2014), Hefei, Anhui, China, DOI: 10.1109/ICCCNT.2014.6962991, pp. 1-5, July 11-13, 2014.

[17] Jitender Grover, Shikha and Mohit Sharma, "Location Based Protocols in Wireless Sensor Network - A Review", IEEE Fifth International Conference on Computing, Communications and Networking Technologies (ICCCNT 2014), Hefei, Anhui, China, DOI: 10.1109/ICCCNT.2014.6962990, pp. 1-5, July 11-13, 2014.

[18] Jitender Grover, Shikha Sharma and Mohit Sharma, "Optimized GAF in Wireless Sensor Network", IEEE 3rd International Conference on Reliability, Infocom Technologies and Optimization (ICRITO 2014), Amity University, Noida, DOI: 10.1109/ICRITO.2014.7014686, pp. 01-06, October 8-10, 2014.

[19] Jitender Grover, Shikha Sharma and Mohit Sharma, "Reliable SPIN in Wireless Sensor Network", IEEE 3rd International Conference on Reliability, Infocom Technologies and Optimization (ICRITO 2014), Amity University, Noida, DOI: 10.1109/ICRITO.2014.7014694, pp. 01-06, October 8-10, 2014.

[20] A. El-Hoiydi and J. Decotignie, Low power downlink mac protocols for infrastructure wireless sensor networks, ACM Mobile Networks and Applications, vol. 10 (5), pp. 675-690, 2005

[21] Pankaj Pardesi and Jitender Grover, "Improved Multiple Sink Placement Strategy in Wireless Sensor Networks", 2015 IEEE International Conference on Futuristic Trends on Computational Analysis and Knowledge Management (A-BLAZE), Amity University, Greater Noida, Uttar Pradesh, India, DOI: 10.1109/ABLAZE.2015.7155032, pp. 418-424, 25-27 Feb, 2015.

[22] Jitender Grover and Anjali, "Wireless Sensor Network in Railway Signalling System", The IEEE International Conference on Communication Systems and Network Technologies (CSNT-2015), Shri Ram Group of Institutes, Gwalior, DOI10.1109/CSNT.2015.28, pp. 308-313, April 04-06, 2015.

[23] Renu Sharma and Jitender Grover, "Mitigation of Byzantine attack using Enhanced Cooperative Bait Detection and Prevention Scheme (ECBDPS)", 4th IEEE International Conference on Reliability, Infocom Technologies and Optimization (ICRITO-2015), Amity University, Noida, DOI: 10.1109/ICRITO.2015.7359296, pp. 1-6, September 2-4, 2015.

[24] Avinash More, Vijay Raisinghani, Random Backoff Sleep Protocol for Energy Efficient Coverage in Wireless Sensor Networks, Smart innovation,System and Technology,Springer,Varlag, vol. 28 (2), pp. 323-331, 2014.

[25] Michael Buettner, Gary V. Yee, Eric Anderson, and Richard Han, X-MAC: A Short Preamble MAC Protocol for Duty-Cycled Wireless Sensor Networks, in Proceedings of the 4th International Conference on Embedded Networked Sensor System, pp. 307$320,2006$. 